\section{Males diarreicos en la costa ecuatoriana: cambios socioambientales y concepciones de salud}

\author{
Diarrheal illnesses on the Ecuadorian coast: \\ socio-environmental changes and \\ health concepts
}

\author{
1 Anthropology Department, \\ Trinity College, Hartford, \\ U.S.A. \\ 2 Facultad Latinoamericana \\ de Ciencias Sociales - Sede \\ Ecuador, Quito, Ecuador. \\ 3 Pontificia Universidad \\ Católica del Ecuador - Sede \\ Esmeraldas, Esmeraldas, \\ Ecuador. \\ Correspondencia \\ J. A. Trostle \\ Anthropology Department, \\ Trinity College. \\ 300 Summit Street, Hartford, \\ CT 06106-3100, U.S.A \\ james.trostle@trincoll.edu
}

\begin{abstract}
The authors present an ethnoepidemiological study of diarrheal illnesses in 21 communities on the northern coast of Ecuador, where numerous social and environmental changes have taken place since 2001 due to a new highway. As communities realize that nature itself is changing, changes occur in their interpretations of health and disease, which the authors present through a taxonomic classification of diarrheal illnesses. Given the high incidence of diarrheal diseases, alternative concepts have emerged (as compared to those of biomedicine) in relation to causes, symptoms, and treatments. The non-biomedical and biomedical systems overlap, with mixtures of coexistence and resistance. Recognizing this reality means understanding a series of challenges for the official health system, including the indiscriminate use of antibiotics, non-use of health services for some diseases, and perceived relations between environmental contamination and the efficacy of modern and traditional medicines.
\end{abstract}

Diarrhea; Health Services; Ethnography
James A. Trostle 1

Jeanneth Alexandra Yépez-Montufar 2 Betty Corozo-Angulo 3

Marylin Rodríguez ${ }^{1}$

\section{Introducción}

Las enfermedades diarreicas aún son importantes fuentes de morbilidad y mortalidad en el mundo, sobre todo en niños menores de cinco años 1 . Ante la fuerte incidencia de las enfermedades diarreicas existen concepciones alternativas sobre sus causas, síntomas y tratamientos que circulan al margen del sistema biomédico. En los últimos 30 años varios antropólogos de la medicina han revelado la variabilidad en enfermedades llamadas "tradicionales", y han mostrado esta variabilidad en forma de taxonomías locales de las diarreas 2,3.

Dentro de la salud pública también, algunos investigadores han prestado atención a la medición de las enfermedades diarreicas, tanto desde una perspectiva biomédica, como nobiomédica. Dichas perspectivas no-biomédicas están basadas en tradiciones y prácticas (como curanderismo), existiendo antes de la llegada de la biomedicina, y también contemporáneas (como la medicina naturista o homeopática), pero con supuestos que compiten y/o contradicen los de la biomedicina. Están basadas en los conocimientos locales sobre lo que es un mal, sus causas y posibles tratamientos. En su análisis señalan instancias de traslape o superposición entre conceptos no-biomédicos y conceptos biomédicos 4,5,6,7,8. Cuando esto ha tenido un carácter poblacional, ha sido llamado "epidemiología local”, "etnoepidemiología” 9,10,11,12, o epidemio- 
logía cultural 13. En lugares donde la población local está pasando por grandes cambios en la vida social, uno puede ver con aún más claridad cómo estos sistemas van evolucionando conjuntamente 14,15,16.

Esta investigación describe una etnoepidemiología de las enfermedades diarreicas en la costa norte del Ecuador, zona en la cual hay muchos cambios desde el 2001 por la presencia de una carretera pavimentada, en una región que hasta el 1994 sólo tenía acceso a otros lugares por mar y río. La llegada de la carretera sirve como ejemplo de lo que pasa en muchos lugares donde hay transformaciones abruptas en el espacio natural y los estilos de vida predominantes. En este proceso se van alterando y negociando los nuevos y viejos conceptos sobre la salud y la enfermedad.

\section{Escenario}

Hemos investigado 21 pueblos de la provincia de Esmeraldas en la costa norte del Ecuador, en su mayoría afroecuatorianos. Estos pueblos fueron escogidos aleatoriamente de entre los más de 150 pueblos de los cantones Eloy Alfaro y San Lorenzo. Los cantones forman parte del área de bosque lluvioso tropical llamado "El Gran Choco", o el "Chocó biogeográfico del Pacífico" que cubre parte de las costas del Ecuador, Colombia y Panamá. Este área ha sido catalogada como un "biological hotspot" 17, es decir, una zona en la cual una concentración extraordinaria de especies están perdiendo una cantidad extraordinaria de su "hábitat". Los pueblos bajo investigación están ubicados en las carreteras de la región y también en los márgenes de los ríos Cayapas, Santiago y Onzole, y en una zona de manglares cerca de la costa, llamada "Bajo Borbón" (Figura 1). Los ríos mencionados desembocan en Borbón, que es el pueblo más grande del área con aproximadamente 5,000 habitantes. De acuerdo al censo nacional ecuatoriano del 2001, casi un 55\% de la población de los cantones Eloy Alfaro y San Lorenzo se autoidentifican como afroecuatorianos y un 13\% como Chachi, el grupo indígena que predomina en la región 18 . Los demás se autoidentifican como mestizos, mulatos, o blancos, categorías que apuntan a tendencias migratorias recientes por la llegada de blancos y mestizos provenientes de otras provincias 19. Aunque hay proporciones mayoritarias de afroecuatorianos en los pueblos del estudio, hay variabilidad en otros aspectos. Por ejemplo, comparando entre los 21 pueblos y dentro de los mayores de 13 años, el porcentaje de personas viviendo que nacieron, y continúan residiendo en el mismo pueblo varía entre $0 \%$ y
79\%; el promedio de estadía varía entre 7,2 y 33 años; y el promedio de años de educación varía entre 3,1 y 6,0 20. En general, los pueblos lejanos tienen mayores porcentajes de afroecuatorianos, mayores proporciones de residentes nacidos en el mismo pueblo, y mayores promedios de estadía en el pueblo.

La región cuenta con un hospital regional en Borbón, seis subcentros de salud rurales y seis puestos de salud estatales que se encuentran dispersos en los ríos y suelen tomar la función de recurso de emergencia. Pero sólo cinco de las 21 comunidades cuentan con puestos de salud atendidos por una auxiliar de enfermería y los médicos funcionan bajo un calendario rotativo propuesto por el Ministerio de Salud.

De acuerdo con estudios regionales hechos por el Ministerio de Salud Pública del Ecuador, las principales causas de morbilidad en la Provincia de Esmeraldas son infecciones respiratorias, enfermedades diarreicas, hipertensión arterial, enfermedades venéreas, diabetes, paludismo, y dengue 21. Los dos cantones de este estudio han sido nombrados entre los 15 más vulnerables de los 226 cantones en Ecuador, incluyendo recursos del agua y saneamiento, salud, educación, pobreza, y población agrícola 22 .

\section{Métodos}

Este estudio multidisciplinario fue diseñado por un epidemiólogo y un antropólogo. Los componentes metodológicos centrales son:

a) Vigilancia activa de síntomas asociados con las enfermedades diarreicas (todo el año, durante 4 años, por parte de los promotores de salud que viven en o cerca de cada pueblo en el estudio);

b) Un estudio caso-control que visita cada pueblo cada 7 meses por 15 días, durante el cual hay identificación de casos de diarrea, selección de controles dentro del hogar y en la comunidad, toma de muestras de heces y aplicación de varias encuestas;

c) Un estudio geográfico que mapea los ríos, los pueblos y las carreteras, incluyendo las casas, letrinas, fuentes de agua, etc., como parte de un sistema de información geográfica;

d) Estudios microbiológicos que identifican los agentes patógenos en la zona y sus niveles de resistencia a antibióticos; y

e) Varios estudios etnográficos y sociológicos que incluyen censos anuales de la población, estudios de redes sociales en cada pueblo, y una serie de entrevistas individuales y grupales junto con observaciones puntuales.

Los resultados incluidos aquí provienen sobre todo de los estudios etnográficos y sociológicos. 
Figura 1

Mapa de la región. Provincia de Esmeraldas, costa norte del Ecuador.
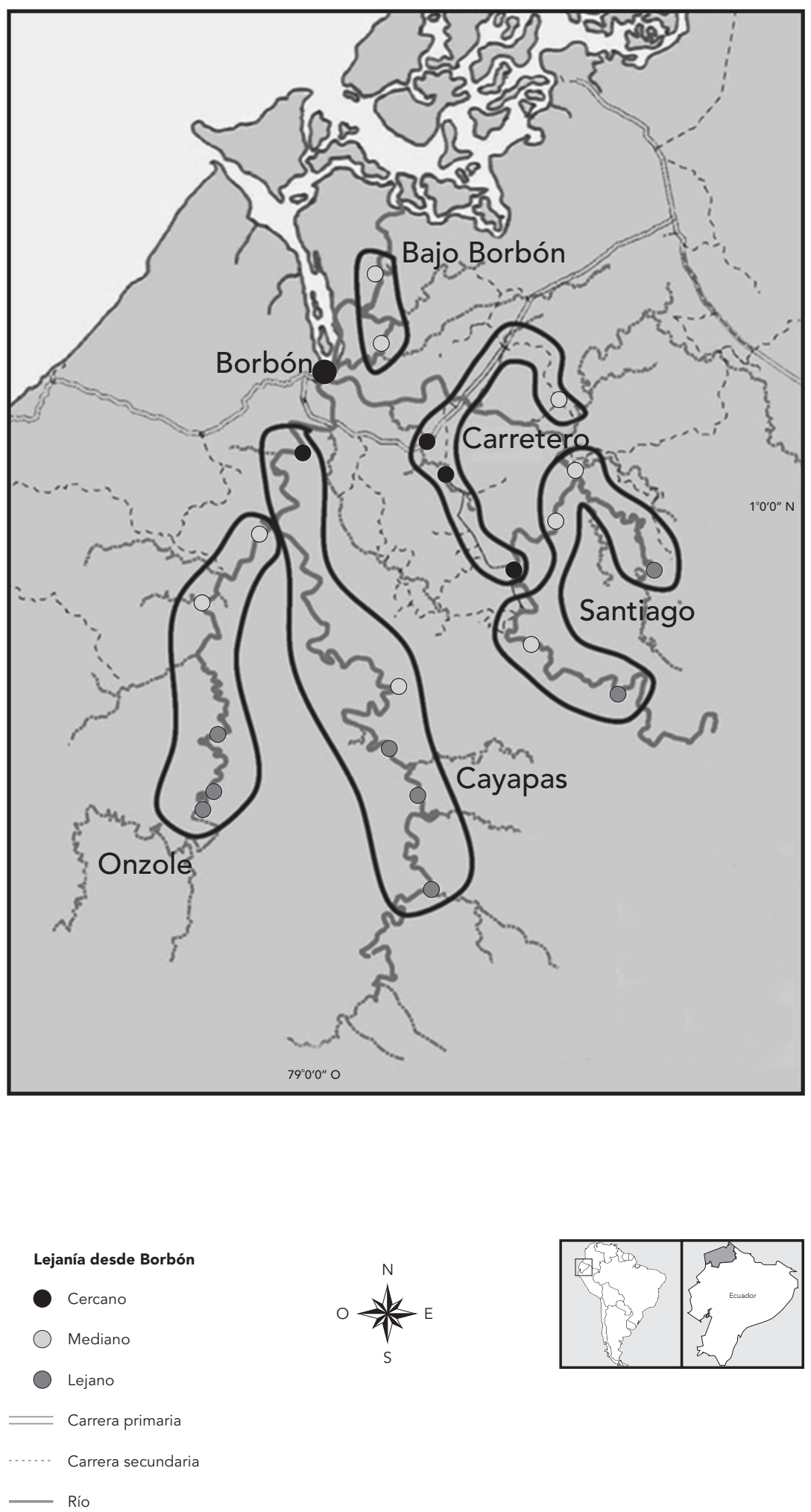
Una antropóloga visitó cada pueblo, junto al equipo caso control, en los primeros 3 años del estudio, y en los 2 próximos años ocasionalmente con el equipo caso control y en ocasiones sola. También hubo estudiantes que pasaron entre 8 y 10 semanas cada verano haciendo estudios etnográficos. En total, unas 128 semanas fueron pasadas en los pueblos por estas antropólogas. Las antropólogas tomaron notas basadas en sus entrevistas y observaciones, y además hicieron 227 entrevistas formales, grabadas y transcritas. También se realizó un registro fotográfico.

La información sobre diagnósticos, tratamientos, e ideas sobre las enfermedades diarreicas están basadas en observaciones y conversaciones informales, y entrevistas con madres, cuidadores de niños en general, y agentes de salud profesionales y no-profesionales, tales como promotores, médicos, enfermeras, curanderos, parteras, y otras. Nuestro objetivo principal para las entrevistas y observaciones era captar cómo la gente entiende, explica y trata las enfermedades diarreicas de conocimiento común en la región. Editamos algunos de los comentarios para aumentar su claridad.

Los procedimientos de este estudio fueron revisados y aprobados por los Comités de Ética de la Universidad San Francisco de Quito (Ecuador); de Trinity College (EEUU); y de la Universidad de Michigan (EEUU). Todos los participantes dieron su consentimiento informado.

\section{Análisis}

Todos las notas y transcripciones (447 documentos textuales de 1 a 30 páginas) fueron incluidas en una base de datos cualitativos (ATLAS. ti; http://www.atlasti.com), donde fueron codificadas usando 213 códigos desarrollados para captar los distintos temas y objetivos del proyecto. Utilizamos los códigos para agrupar la información por categorías. Se crearon, por ejemplo, códigos que captaron la mezcla de medicinas y tratamientos provenientes de diversas tradiciones medicinales. Al desarrollar las descripciones de enfermedades y de tratamientos, regresamos a los pueblos para asegurar que nuestras aproximaciones fueran entendibles y aceptables por parte de la población local. Hubo un taller de validación con los 25 promotores de salud de los pueblos para discutir los resultados presentados en este manuscrito.

\section{Resultados}

\section{Taxonomía de la variabilidad}

en males diarreicos

La Tabla 1 es un resumen generalizado que demuestra la pluralidad en nombres y tratamientos usados para males diarreicos en la zona.

Las explicaciones locales sobre las causas y el tratamiento adecuado para cada tipo de diarrea muestran una compleja sistematización de factores como: la edad del paciente, las características físicas de la diarrea o los sucesos que antecedieron el padecimiento. La taxonomía también demuestra las mixturas de síntomas y causas usadas para definir distintos males. Algunos pueden o no tener diarrea como síntoma (ojo, los espantos, malaire, almorranas), mientras los demás son caracterizados sólo por la presencia de la diarrea. El apestoso olor de la diarrea es asociado más con malaire que con los otros males, el color verde es asociado con las heces de la diarrea por sereno, y las heces con sangre son un síntoma definitivo de las almorranas. El mal de ojo y espanto también son asociados con fiebre y vómito, y falta de apetito, y el malaire es asociado con todos estos síntomas menos con la fiebre. Las enfermedades diarreicas por sereno y colerín son las únicas que afectan a niños menores de cinco años, pues la causa del sereno son las prendas de vestir que utiliza un/a niño/a expuesta al ambiente o aire libre después de las 18:00hrs. y la del colerín es la ingesta de leche materna de una madre que ha estado enfurecida.

Las causas de estos males se pueden atribuir a la mirada fuerte de una persona que siente simpatía o antipatía por una persona, como en el caso del ojo. El espanto seco y espanto de agua también sucede a causa de fuertes impresiones en la vida diaria. El malaire, en contraste, es causado por la presencia de espíritus que están en el ambiente, y el sereno también es resultado de la exposición a momentos de transición entre la luz y la oscuridad. Las almorranas no tienen causa definitiva, aunque varios piensan que tienen algo de ver con el consumo de comida mala. Algunos otros males diarreicos son productos de comportamientos relevantes a la comida y a la higiene, como comer en exceso (indigestión) o condiciones higiénicas del ambiente (bicho/parásito o escorbuto o infección).

El ojo es la única enfermedad que, siendo diagnosticada por un curandero, ni merece ni puede recibir ningún tratamiento biomédico. Puede ser hasta contraproducente recibir tratamiento biomédico. En ese sentido es uno de los pocos males que resiste la inclusión en las 
Tabla 1

Taxonomía de males diarreicos.

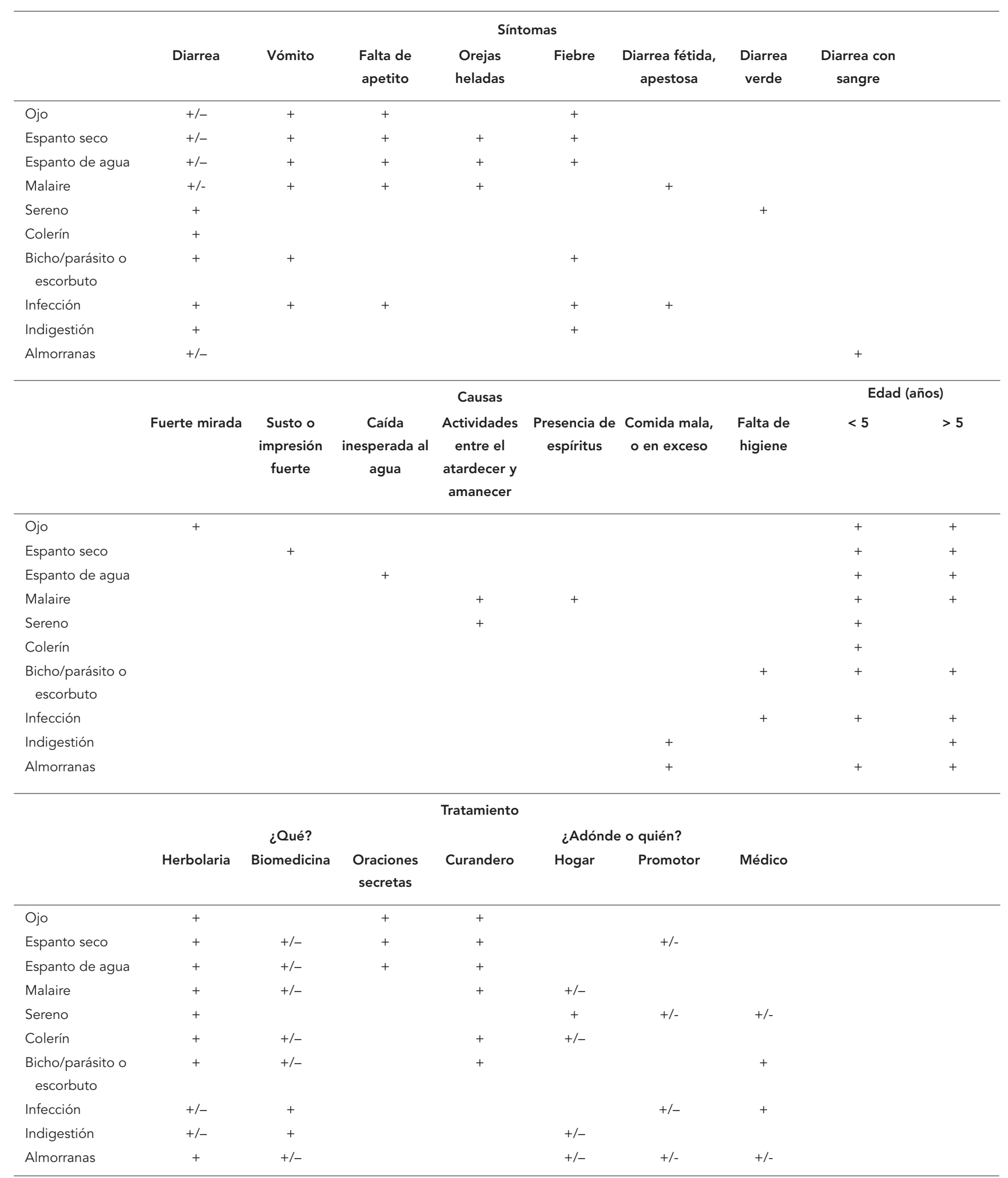

Clave: "+" = relevante siempre; "+/-" = relevante a veces. 
llamadas "mixturas" de tratamiento biomédico y tradicional.

Dados los detalles sobre causas y tratamientos, se puede concluir que, en cuanto a las enfermedades diarreicas, los habitantes en los cantones Eloy Alfaro y San Lorenzo sienten que viven en un mundo donde pueden ser amenazados por comportamientos humanos (nadar, caer, pasar tiempo en el bosque, comer demasiado), por emociones y relaciones sociales (envidiar, asustar, enojar), o por exposición a los espíritus o fuerzas naturales como la luna. Pero también los habitantes están amenazados por exposición a otros agentes pequeños, que viven en el mismo medio ambiente, llamados bichos/parásitos o escorbuto, y también por la llegada de nuevos "agentes patógenos" invisibles, interpretados como infecciones.

Estos son ejemplos de la sistematización y complejidad de las enfermedades locales. Se puede leer la Tabla 1 como un espectro de clasificaciones de males diarreicos, desde las basadas en comportamientos, emociones, espíritus, o fuerzas naturales, hasta las basadas en un mundo invisible e interior de bichos e infecciones, algunos solamente visibles a los médicos con sus pruebas de heces y sangre. Las personas han desarrollado conceptos que se explican y se solucionan por medio de su concepción de lo que es un mal y sus causas.

\section{Mixturas entre sistemas de salud}

La taxonomía también demuestra las mixturas de tratamientos utilizables para estos males. (Usamos la palabra mixtura para enfatizar que la búsqueda de alivio es un proceso activo, y que no es siempre fiel a una u otra tradición de medicina, ni son los mismos practicantes siempre fieles a sus propias tradiciones). Todos los males, menos el ojo, pueden tratarse con hierbas o tratamientos biomédicos. Los servicios médicos locales no siempre son una opción satisfactoria, ya que carecen de medicamentos y el personal capacitado para atender cualquier clase de enfermedades o males. Pero las decisiones sobre tratamientos no están basadas solamente en la disponibilidad o calidad de los servicios, sino en las concepciones de ambas partes (proveedores y usuarios) sobre lo que son las enfermedades diarreicas y de dónde vienen.

Para algunos médicos sus concepciones sobre los males diarreicos se asemejan a las de los pobladores: "es algo a lo que hay que acostumbrarse..., por ejemplo, cuando una persona ha llegado con una enfermedad diarreica y yo he recetado algún medicamento ha habido ocasiones en que la persona no se lo toma, pues aducen que tienen ojo; en otra ocasión vino una mujer quejándose de un fuerte dolor de cabeza. Yo le ofrecí unas pastillas para el dolor pero eso fue todo. Luego de unos días esta persona empeoró y me dijo que visitaría a un curandero, pues sospechaba que tenía mal aire. Al día siguiente yo mismo pude constatar como la paciente se restableció después de visitar al curandero..." (médico rural).

Puesto que las personas tienen varios males asociados con la diarrea, antes de realizar cualquier diagnosis los médicos prefieren preguntar primero si los pacientes ya han sido tratados por ojo, espanto o mal aire. De igual manera, cuando las personas han tenido acceso a un médico antes que a un curandero, se muestran reticentes a aceptar tratamiento por un mal diarreico, sobre todo en el caso de la diarrea por ojo, ya que se comenta sobre casos en que las personas murieron cuando se les administró algún tipo de medicamento (inyecciones o suero) al tener ojo (comparar 23,24,25). Aunque los médicos siguen manteniendo una postura de escepticismo frente a la interpretación de los males diarreicos en esta zona, han tenido que moderar sus prácticas y reconocer la vigencia activa del sistema de la herbolaria y curanderismo comunes en estos poblados.

Lo propio ocurre con las curanderas/os y remedieras con respecto a la biomedicina. Aunque muchos de estos agentes de salud son los portadores de una larga tradición muy apreciada en esta zona, ellos reconocen "que las enfermedades de ahora ya no son las mismas que las de antes. Antes usted se tomaba una hierba y se curaba pero ahora necesita una pastilla (...) La única enfermedad en que no puedo dar pastilla es el ojo porque ahí si una persona recibe medicamento se puede morir" (curandera, 56 años).

En forma semejante, los promotores de salud reconocen la coexistencia de varias formas de explicación de las causas de enfermedades diarreicas en la región: "estamos apuntando en lo cultural, lo autóctono, lo que realmente hay dentro de nuestro convivir diario. Cuando ya hablamos de la infección lo asociamos, porque ya tenemos el conocimiento frente a una diarrea, que por el olor, por la característica también lo conjugamos. Además de tener mal aire, además de ser espanto, ya lo catalogamos como una infección intestinal, eso ya en base a lo ya que hemos aprendido" (promotor de salud, 60 años).

En el caso de los males diarreicos, las decisiones complejas no solamente están en manos de médicos, curanderos o promotores, sino que son parte del "sentido común de todas las mujeres de acá. Todas saben manejar sus hierbas y curar a sus hijos de alguna diarrea" (poblador, 45 años). Unos de los ejemplos más comunes para "tran- 
zar" (cortar, parar) la diarrea en niños y adultos, son las mezclas de terramicina con jugo de limón, la infusión de diescansel con Imodium, o el Alka-Seltzer con jugo de limón.

En los casos en que no existe acceso a ningún medicamento, constatamos el uso casi generalizado del jugo de limón. Por ejemplo, una mujer de unos 38 años dijo: "otra cosa que si hacemos que cuando está la fiebre muy alta se recomienda el limón en vez del jarabe o la pastilla. Mi abuelita me enseñó a coger el limón, luego exprimirlo en una bandeja con un poquito de agua y sobarse todo el cuerpo. Con ese limón baja la fiebre y la gripe. Si tiene gripe también se mejora un poco, porque no quita sólo mejora. Ayuda aflojar la flema tomar la limonada hervida y es bueno para el dengue y la tifoidea, eso sí lo tengo muy presente".

Sobre el uso del jugo de limón para la diarrea, otra mujer de más de 60 años nos dijo que “lo que se acostumbra es darle agua de limón y eso hace que se le suspenda". Lo que se deduce es que el limón es la combinatoria principal porque se le atribuye el poder de "cortar" la diarrea.

Los males como el sereno y el colerín, que solamente atacan a los niños menores de cinco años, suelen ser detectados en primera instancia por las madres. En el caso del sereno, ellas mismas aplican la cura mientras que si se trata de colerín, remiten al niño a la curandera. En gran parte los recursos de la herbolaria local tienen que ser agotados antes de recurrir al médico, por el costo y tiempo de viaje que existe entre muchas de las comunidades y los centros hospitalarios.

"Cuando alguien se enferma se busca primero al curandero; aquí tenemos a don 'Pedro' que cura ojo, espanto, mal aire, también estaba doña 'Juana' ahora ya no ejerce esta profesión. Después se lleva al paciente, si no se le ve mejoría con la medicina natural, donde la promotora que cuando tiene medicamento le receta al paciente después de preguntarles las dolencias que tiene. Pero si no se mejora se lo lleva a Borbón" (hombre, 56 años).

Sin embargo, el acceso a hierbas, plantas medicinales y curanderos no es el mismo en todas las comunidades. En una de ellas, por ejemplo, se ha observado que los químicos como el Glifosato que utilizan para eliminar el monte, ha terminado con todas las plantas medicinales. Esta escasez impide el uso adecuado de los tratamientos locales. Así mismo, en otras comunidades la introducción de la carretera ha cambiado la práctica de las mujeres por tener sus propios huertos, porque ahora dependen de los vendedores ambulantes que llegan a vender legumbres y vegetales.

La disponibilidad de productos medicinales y medicamentos varía también en la zona. En una encuesta que hicimos en dos distintas ocasiones contando la disponibilidad de tratamientos para enfermedades diarreicas hubo disponibilidad de antibióticos en 16 de 19 pueblos, hubo disponibilidad de antidiarreicos en 11 de 19 pueblos, antiparasitarios en 15 de 19 pueblos y suero oral en sólo 6 de 19 pueblos.

Pudimos ver la existencia de diversas fuentes de productos del sistema biomédico, conviviendo con la herbolaria nativa de la zona y con el comercio ambulante de productos naturistas. Por ejemplo, hemos visto el uso de calas fabricadas con hierbas como tratamiento para la diarrea y curanderos recomendando el uso de infusiones de plantas para mejorar la efectividad de ciertos analgésicos. También existe una amplia gama de matices en el acceso a las medicinas, sobre todo cuando las personas tuvieron que enfrentarse con los males diarreicos en unos poblados más remotos que otros. El testimonio de uno de las promotoras de salud de una comunidad remota nos explica: “...entre nuestra gente todavía sobreviven las tradiciones antiguas. Se utilizan mucho las hierbas apagadas [a infusión] con tetraciclina o terramicina, cuando hay en la tienda. En nuestra gente... todavía están con las tradiciones antiguas mas se les da hierbitas apagadas que la tetraciclina. La tetraciclina yo, en mi pequeño botiquín, no la uso y aquí mi hijo también con mi nuera tienen su tiendita tampoco no veo que ellos tengan la tetraciclina, ni la terramicina, porque eso va que las personas a veces se automedican y al niño le hacen un daño. A veces usted ve bastantes niños con la dentadura mala por que usan mucha la tetraciclina, la terramicina".

La presencia de una enfermedad diarreica en estas comunidades es un desafío. Para responder a él, no siempre se buscan respuestas absolutas, sino opciones que transitan a través de las porosidades visibles entre el sistema biomédico y los otros sistemas de salud. Estas mixturas reflejan los cambios grandes que hay en esta región. La búsqueda de servicios tradicionales o biomédicos se ha expandido a través de la apertura de caminos que han facilitado los muchos cambios medioambientales y socioeconómicos en la región. Como veremos abajo, estos cambios están afectando la forma en la cual la gente entiende lo que es la naturaleza, su fuerza y su relevancia como fuente de sustancias saludables.

\section{Principales cambios medioambientales y socioeconómicos y sus implicaciones para la salud}

La fuente de muchos cambios contemporáneos en la región es una carretera pavimentada que va desde Borbón hacia la costa (terminada en 1996), y desde Borbón hacia los Andes (terminada en 
2001). Estas carreteras han reducido en un $50 \%$ (de 12 a 6 horas) el tiempo de viaje desde Quito a Borbón. Solo 4 de los 21 pueblos fueron accesibles por carretera durante la mayoría de esta investigación (de 2002 a 2006), pero los productos que han llegado por carretera llegan a todos los demás por río. Las carreteras y caminos han abierto la región a nuevas formas de producción, sobre todo extracción de madera y plantaciones de la palma africana 26 .

El bosque lluvioso-tropical donde están ubicadas las 21 comunidades del estudio es el principal recurso y fuente de orgullo para los pobladores de la zona. La explotación maderera y minera tiene una historia de más de un siglo, que, sin embargo, se ha intensificado en las últimas tres décadas por el ingreso de maquinaria pesada a la zona. También se ha formado una zona de plantaciones de la palma africana, que también ha contribuido a la pérdida del bosque. Se estima que entre 1995 y 2005 se deforestó de 135.000ha a 180.000ha en el norte de Esmeraldas, prácticamente el doble que en la década anterior 27 . Estos sucesos han generado serios cambios en los sistemas económico laborales y medioambientales en la región.

Aunque la gratificación económica inmediata es uno de los beneficios principales de dichas actividades, la contaminación de los ríos y los cambios en el medio ambiente se han hecho evidentes. Las quejas de los pobladores sobre las consecuencias ambientales varían desde la contaminación del agua por el transporte de la madera por los ríos, a la falta de animales de monte para cazar, y la sequía de las vertientes de agua. Por ejemplo, "en el río ya casi no se encuentra nada quizás sea por la lejía de la madera y el combustible de las máquinas. Eso también nos debe estar enfermando a nosotros porque hay ciertos lugares donde cruzan las maquinarias a veces trabajan dentro del rió. Todo eso es contaminación" (hombre, 38 años).

Estas nuevas amenazas hacia la salud de los pobladores, al igual que los nuevos hábitos alimenticios, que también se dan a raíz de la adopción de nuevas formas de trabajo, están cambiando las formas de explicar y tratar las enfermedades diarreicas. Como veremos a continuación estas nuevas formas de trabajo, además de estar cambiando el ambiente físico y social, también están introduciendo nuevos medios de información, los cuales están ampliando el entendimiento local de las enfermedades diarreicas.
Principales cambios en canales de comunicación con respeto a la salud.

La accesibilidad vial ha aumentado las tasas de migración, ha traído nuevos productos relevantes para la salud y la nutrición, y ha facilitado el movimiento de ideas y noticias sobre la salud y la enfermedad. Algunos de estos cambios son bastante palpables. Los movimientos migratorios y la apertura de las carreteras han abierto nuevas formas de comunicación. Por ejemplo, en el 2003 no hubo ningún servicio celular en la zona, mientras que en el 2008 hubo servicio en 8 de los 21 pueblos del estudio. También, en los pueblos más remotos entre el 2004 y 2006, el porcentaje de hogares con televisores creció desde $8 \%$ a $20 \%$, y en el pueblo rural más grande se instaló un equipo de internet por primera vez en el 2007.

Todos estos cambios medioambientales en la zona traen consigo nuevas aspiraciones con respecto a su salud. Durante nuestra investigación, observamos al menos tres canales de comunicación por los que fluyen datos de información. El primer canal es el del sistema biomédico oficial, ya sea por medio de los servicios de salud gubernamentales o no gubernamentales. La información en esta vía, se refiere principalmente a los cuidados preventivos y al tratamiento de enfermedades diarreicas, anémicas, infecciones respiratorias, malaria y oncocercosis. Otros mensajes importantes tienen que ver con procedimientos para la purificación del agua, hábitos higiénicos y el cuidado prenatal. Durante 2007 se observó también la diseminación de proyectos para el reciclaje y tratamiento de la basura, sobre todo en los ríos Onzole y Wimbí.

La segunda ruta es la de los medios de comunicación. Aunque no todas las familias son propietarias de un televisor o de una radio, es una costumbre entre los pobladores permitir la presencia de todos quienes deseen compartir un programa o película, a pesar de la escasez del servicio de luz eléctrica o su deficiente calidad. En los programas televisivos hay una ausencia casi total de temas de higiene y salud que tengan un contenido relevante para la realidad socio cultural de la zona. Lo que más se escucha, son los anuncios comerciales en los que se publicitan diversos tipos de medicamentos y "pastillas" naturistas y programas radiales matutinos que hablan de eventos milagrosos, logrados a través del uso de ciertos productos "naturales".

Toda esta información se decanta finalmente en las conversaciones diarias, especialmente entre las mujeres de cada poblado. Estos diálogos tienden a hacer referencia al conocimiento de la herbolaria, consejos cotidianos para el cuidado 
de los niños y los ancianos y experiencias con respecto al uso de medicamentos. Otros conocimientos incorporados sobre nuevas plantas, hierbas o medicinas, pueden provenir de los viajes entre ríos o poblados, de los viajes hacia las ciudades o centros urbanos o de la migración permanente que forman parte de los cambios socioeconómicos de la zona.

Cabe mencionar la influencia que tienen las percepciones y experiencias de las mujeres afroecuatorianas que se emplean en el servicio doméstico en las ciudades de Quito, Guayaquil y Esmeraldas. Esta refleja a la vez la importancia del mercado laboral en empujar migración hacia y fuera de los pueblos rurales, pero también la importancia de estos movimientos como una fuente de nuevos conocimientos sobre la salud. Una de las mujeres de la zona que colaboró con nuestro estudio, tenía en su casa una botella de tónico cerebral, supuestamente elaborado a base de "hiel de hígado de rana". Durante su estancia de trabajo como cocinera en una casa situada en un elegante barrio Guayaquileño, ella observó como su patrona utilizaba un jarabe naturista para que su hijo rindiera mejor en la escuela. Estimulada por esta observación, ella adquirió el mencionado tónico a un vendedor ambulante, con la esperanza de que sus niños también mejoraran sus calificaciones escolares. "Mi hijo no va bien en clases y yo quiero que vaya bien en las materias. Yo no le puedo ayudar mucho porque yo no pasé por la escuela, pero yo le compro esos jarabes para que le hagan crecer la cabeza, que pueda estudiar". Para ella la inteligencia y el rendimiento escolar de sus hijos se había incorporado como un nuevo interés relacionado con la salud y bienestar futuro de toda su familia, más allá inclusive de las dificultades en el acceso a la educación que se viven en esta zona y sin cuestionar tampoco los componentes o la efectividad del producto adquirido.

Las concepciones que se tienen en esta región acerca de la salud y la enfermedad, van mostrando unos cambios cuya complejidad y variabilidad también afecta a las enfermedades diarreicas o a los males que guardan relación con dichas enfermedades. Como nos dijo una mujer de unos 50 años, hablando sobre diferencias en el manejo de la diarrea: "bueno, o sea ellos lo que esperan es que, de curarse pues con el medicamento, a más que todo en la mayoría de las mamás, ellas creen más en el medicamento que en las hierbas, porque el problema creo que... quizás también no saben usarla no, pero siempre si un niño está con una diarrea, un dolorcito de barriga ellas no le dan a veces nada, sino que lo que primero acuden al subcentro más que todo en las jóvenes".

\section{Conclusión}

La inclusión, competencia, hibrideces y/o mixtura de explicaciones biomédicas (como la diarrea tal cual, o infección, o bichos) y también tradicionales (como el ojo o espanto) para males diarreicos es algo visto en muchos lugares en el mundo 3,5,7,8,11. Fue visto en esta misma área geográfica unos 20 años antes del comienzo de nuestro estudio: en 1983, Pederson \& Coloma 24 describieron disponibilidad común de recursos tradicionales, empezando a mezclarse con recursos biomédicos. También Quiroga 28 describe la fuerte importancia de los curanderos en la búsqueda de alivio o cura en Muisne, una zona al sur de la nuestra. Es claro que estas ideas tienen siglos de tiempo de circulación en la región, y que reflejan una larga historia de intentos de diagnosticar, tratar y prevenir las amenazas naturales y espirituales que la gente enfrenta, y que ahora pueden ser llamados "males diarreicos".

Lo innovador de este estudio es ver cómo las relaciones entre estas dos explicaciones están evolucionando en un periodo de corto plazo, bajo presiones de cambios sociales abruptos y drásticos. En una región donde antes hubo muchos curanderos y pocos médicos, ahora hay ambos agentes; además de naturistas 29 y promotores y libre acceso a muchos productos farmacéuticos. Además de explicaciones para enfermedades basadas en espíritus o basadas en microbios, también la gente construye explicaciones basadas en cambios en el mundo exterior (contaminación ambiental, basada en el poder de tratamientos herbolarios), y basadas en ideas sobre debilidades físicas (falta de vitaminas, efectos nocivos del estrés). Los residentes de esta región están enfrentando a la vez una amplia gama de enfermedades nuevas como el cólera o el dengue, y también una amplia gama de posibles sustancias de prevención o tratamiento. Y los agentes de la salud están adaptando sus prácticas a este ambiente, creando nuevas prácticas que son mezclas de distintas tradiciones. Las transiciones sociales a raíz de los cambios ambientales y económicos, son la causa que atribuimos a la variedad de intercambios entre lo no-biomédico y biomédico dentro de la taxonomía.

La coevolución de las prácticas (biomedicina, herbolaria, curanderismo, medicina naturista) se expresa debido a los cambios socioambientales en la zona del estudio. Estos cambios han conllevado a la flexibilidad de algunos médicos escépticos de respetar algunas de las prácticas históricas de los nativos. Éstos a su vez acceden a la introducción de una serie de nuevas prácticas y productos que en muchas localidades está mez- 
clando y reemplazando las prácticas tradicionales. Los impactos ecológicos fuertes y negativos conllevan a la población local a la búsqueda de servicios de salud que incluyen mixturas de coexistencia (en formas de tratamiento para curar sobre todo enfermedades diarreicas).

La epidemiología es, a la vez, relevante para síntomas clasificados como biomédicos y otros clasificados como locales o tradicionales. Dada que es, en parte, una descripción del peso de la enfermedad, se puede presentar este peso como una epidemiologia clásica, buscando cifras de in- cidencia y prevalencia y factores de riesgo para el ojo o la diarrea (ver por ejemplo, esta hecha con nervios y su traducción a diagnósticos psicológicos en Puerto Rico ${ }^{30}$ ). No obstante, también puede presentar este peso como una epidemiología sin números, intentando describir la carga de la enfermedad en palabras en vez de números 9,31. Lo que estamos haciendo en este estudio es una exploración de las dos epidemiologías, pensando en su intercambio y evolución mutua, conjunta, en una zona de transiciones sociales y ambientales veloces.

\section{Resumen}

Presentamos una etnoepidemiología de las enfermedades diarreicas en 21 pueblos de la costa norte del Ecuador, zona en la cual hay muchos cambios sociales y ambientales desde 2001 por la presencia de una nueva carretera. Con percepciones de que la misma naturaleza está cambiando, vienen cambios en interpretaciones de lo que es salud y enfermedad, que presentamos en forma de una taxonomía de males diarreicos. Ante la fuerte incidencia de las enfermedades diarreicas, existen concepciones alternativas a la biomedicina en cuanto a sus causas, sintomas y tratamientos. Hay superposiciones entre sistemas no-biomédicas y biomédicos, en donde hay mezclas de coexistencia y resistencia. Reconocer esto es entender una serie de desafíos para el sistema de salud oficial, incluyendo el uso indiscriminado de antibióticos, el no uso de servicios de salud para algunos males, y relaciones percibidas entre contaminación ambiental y la eficacia de las medicinas moderna y tradicional.

Diarrea; Servicios de Salud; Etnografía

\section{Colaboradores}

J. A. Trostle fue responsable por el diseño, recolección de datos, análisis, redacción y aprobación final. J. A. Yépez-Montufar, B. Corozo-Angulo y M. Rodríguez contribuyeron en la recolección de datos, análisis, redacción y aprobación final.

\section{Agradecimientos}

Este estudio fue apoyado por National Institute of Allergy and Infectious Diseases del National Institutes of Health (NIH/NIAID), RO1-AI050038. Los autores agradecen el equipo de campo del Proyecto Ecología, Desarrollo, Salud y Sociedad (EcoDeSS) por su ayuda logística en la recolección de estos datos, y la Asociación de Promotores del Area de Salud Borbón. 


\section{Referencias}

1. Kosek M, Bern C, Guerrant RL. The global burden of diarrheal disease, as estimated from studies published between 1992 and 2000. Bull World Health Organ 2003; 81:197-204.

2. McKee L. Ethnomedical treatment of children's diarrheal illnesses in the highlands of Ecuador. Soc Sci Med 1987; 25:1147-55.

3. Weiss MG. Cultural models of diarrheal illness: conceptual framework and review. Soc Sci Med 1988; 27:5-16.

4. Heckler SL. Herbalism, home gardens, and hybridization: Wõthïhã medicine and cultural change. Med Anthropol Q 2007; 21:41-63.

5. Iyun BF, Oke EA. Ecological and cultural barriers to treatment of childhood diarrhea in riverine areas of Ondo State, Nigeria. Soc Sci Med 2000; 50:953-64.

6. Bierlich B. Sacrifice, plants, and western pharmaceuticals: money and health care in northern Ghana. Med Anthropol Q 1999; 13:316-37.

7. Granich R, Cantwell MF, Long K, Maldonado Y, Parsonnet J. Patterns of health seeking behavior during episodes of childhood diarrhea: a study of Tzotzil-speaking Mayans in the highlands of Chiapas, Mexico. Soc Sci Med 1999; 48:489-95.

8. Gorter AC, Sánchez G, Pauw J, Pérez RM, Sandiford P, Davey Smith G. Childhood diarrhea in rural Nicaragua: beliefs and traditional health practices. Bol Oficina Sanit Panam 1995; 119:377-90.

9. Fernandes RCP. Uma leitura sobre a perspectiva etnoepidemiológica. Ciênc Saúde Coletiva 2003; 8:765-74.

10. Jenkins C, Howard P. The use of ethnography and structured observations in the study of risk factors for the transmission of diarrhea in highland Papua New Guinea. Med Anthropol 1992; 15:1-16.

11. Nichter M. Use of social science research to improve epidemiologic studies of and interventions for diarrhea and dysentery. Rev Infect Dis 1991; 13 Suppl 4:S265-71.

12. Czeresnia D, Gadelha AM, Coimbra Jr. CEA, Stotz EN, Castiel L. Integração entre epidemiologia e antropologia. Hist Ciênc Saúde-Manguinhos 2000; 6:689-705.

13. Trostle JA. Epidemiology and culture. Cambridge: Cambridge University Press; 2005.

14. Larson CP, Saha UR, Islam R, Roy N. Childhood diarrhoea management practices in Bangladesh. Int J Epidemiol 2006; 35:1430-9.

15. Goldman N, Pebley AR, Gragnolati M. Choices about treatment for ARI and diarrhea in rural Guatemala. Soc Sci Med 2002; 55:1693-712.

16. Terra-de-Souza AC, Peterson KE, Andrade FM, Gardner J, Ascherio A. Circumstances of post-neonatal deaths in Ceara, Northeast Brazil: mothers' health care-seeking behaviors during their infants' fatal illness. Soc Sci Med 2000; 51:1675-93.
17. Myers N, Mittermeier RA, Mittermeier CG, da Fonseca GAB, Kent J. Biodiversity hotspots for conservation priorities. Nature 2000; 403:853-8.

18. Instituto Nacional de Estadísticas y Censo. VI Censo de Población y de Vivienda. Quito: Instituto Nacional de Estadísticas y Censo; 2001.

19. Minda P. Identidad y conflicto. Quito: Ediciones Abya Yala; 2002.

20. Trostle J, Hubbard A, Scott J, Cevallos W, Bates SJ, Eisenberg JNS. Raising the level of analysis of foodborne outbreaks: food-sharing networks in rural coastal Ecuador. Epidemiology 2008; 19:384-90.

21. Ministerio de Salud Pública. Diez principales causas de morbilidad según provincias - Ecuador 2007. http:// www.msp.gov.ec/images/mortalidad\% 202007.pdf (accedido el 03/Abr/2010).

22. D'Ercole R, Trujillo M. Amenazas, vulnerabilidad, capacidades y riesgo en el Ecuador: los desastres, un reto para el desarrollo. Quito: Cooperazione Internazionale/Institut de Recherche pour le Développement/Oxfam GB; 2003.

23. Dalmau-Gavilanes F. Magia médica y psicoterapia en el Ecuador: con un anexo de psiquiatría folklórica en la costa ecuatoriana. Guayaquil: Editorial de la Universidad de Guayaquil; 1988.

24. Pedersen D, Coloma C. Traditional medicine in Ecuador: the structure of the non-formal health systems. Soc Sci Med 1983; 17:1249-55.

25. Naranjo M, coordinador. La cultura popular en el Ecuador. Tomo IX: Manabí. Cuenca: CIDAP; 2002.

26. Rival L. The meanings of forest governance in Esmeraldas, Ecuador. Oxford Development Studies 2003; 31:479-501.

27. Minda P. La deforestación en el norte de Esmeraldas (Eloy Alfaro y San Lorenzo). Esmeraldas: Proyecto de Desarrollo Rural de la Provincia de Esmeraldas; 2006.

28. Quiroga D. Saints, virgins, and the devil: witchcraft, magic, and healing in the Northern Coast of Ecuador [Doctoral Dissertation]. Champaign/Urbana: University of Illinois; 1994.

29. Miles A. Radio and the commodification of natural medicine in Ecuador. Soc Sci Med 1998; 47:2127-37.

30. Guarnaccia PJ, Canino G, Rubio-Stipec M, Bravo $\mathrm{M}$. The prevalence of ataques de nervios in the Puerto Rico disaster study. The role of culture in psychiatric epidemiology. J Nerv Ment Dis 1993; 181:157-65.

31. Almeida Filho N. Epidemiologia sem números. Rio de Janeiro: Editora Campus; 1989.

Recibido el 18/Nov/2009

Versión final presentada el 18/May/2010

Aprobado el 19/May/2010 